\title{
Detection of Runway and Obstacles using Electro-optical and Infrared Sensors before Landing
}

\author{
Satish Kumar V., Sudesh K. Kashyap*, and N. Shantha Kumar \\ CSIR-National Aerospace Laboratories, Bangalore-560 017, India \\ *E-mail: sudesh@nal.res.in
}

\begin{abstract}
For safe aircraft operations, detection of runway incursions especially during landing and takeoff is essential. And it is important that such detection technique is capable of detecting the distant objects so that pilot has enough response time to take corrective action. This paper presents techniques to detect runway and runway incursions using electro-optical color camera and medium wave infrared sensor on-board the aircraft during approach for landing. The detection process consists of horizon detection to reduce runway search space in sensor image and then detect runway and obstacles. The information is then presented to the pilot to improve pilot situational awareness. The performance of the proposed techniques are evaluated in flight simulators with simulated images of electro-optical and infrared sensors on-board the aircraft during approach for landing at a distance of 3 nautical miles from runway threshold during day/night and in low visibility CAT II foggy conditions. Effectiveness of the techniques with statistics of runway detection, miss detection and false alarm for different case studies have been provided and discussed.
\end{abstract}

Keywords: Horizon detection, runway search, runway detection, edge detection, template matching, hough transform

\section{INTRODUCTION}

Due to increase in air traffic, the number of runway incursions is rising year after year and is becoming a serious aviation concern. In 1990, the US national transportation safety board (NTSB) has listed runway incursions as one of the top 10 most wanted transportation safety improvements ${ }^{1}$. In 2001, Federal Aviation Administration (FAA) undertook a detailed study on runway incursion with the database of flight operations at US towered airports from 1997 to 2000 to assess the frequency and severity of runway incursions occurrences ${ }^{2}$. The FAA began several initiatives to reduce the number of runway incursions, including an alerting system for air traffic control (ATC), which is relayed via voice communication to the cockpit. National Aeronautics and Space Administration (NASA) and other leading avionics research teams world over are working on runway incursion prevention system (RIPS) to help provide runway incursion alerts to flight crews. The first step in RIPS is to detect runway and runway incursions prior to landing using an onboard sensor or camera. This requires a detection technique that is capable of detecting distant objects in order to give pilot enough response time for corrective action. The detection technique should be robust to aircraft motion and atmospheric illumination conditions to provide round the clock functionality.

Very few references can be cited in the area of automatic detection of runway, runway incursions using onboard camera or sensor images. Cheng-Hua ${ }^{3}$, et al. proposed a technique to detect moving object on runway prior to landing using onboard infrared camera and navigation aid such as global positioning system (GPS). Archer ${ }^{4}$, et al. presented a technique for detection of runway incursions using airborne forward looking infrared (FLIR) sensors such as short wave infrared (SWIR) and long wave infrared (LWIR). White ${ }^{5}$, et al. developed and demonstrated through simulation studies the fusion system for the detection of runway incursions using onboard X-band weather radar and FLIR sensors. Abdul Rahiman ${ }^{6}$, et al. propose a method to detect and track the runway using pattern matching and texture analysis using digital images from cameras onboard the aircraft. Dong ${ }^{7}$, et al. proposed an automatic runway recognition algorithm based on heuristic line extraction. Wang ${ }^{8}$, et al. presents a method that combines Canny edge detection operator with mean filter to get the runway edges detected from aerial images. Aytekin ${ }^{9}$, et al. proposed a runway detection method based on textural properties of the terrain.

This paper presents two novel methods for detection of runway and obstacles on runway from onboard electro-optical (EO) colour camera and medium wave infrared (MWIR) sensor at a distance of approximately 3 nautical miles while the aircraft is approaching for landing. The novelty of these methodologies includes detection of horizon to reduce the runway search area for quick runway detection, runway detection using template matching with dynamically changing template and using edge detection technique, detection of obstacles on runway and display their location and movement on the runway. The onboard EO camera and MWIR sensor are forward-looking and placed at the centre of the aircraft nose focused along the aircraft bore sight. The methodology is evaluated on a flight simulator for detection of static/moving obstacles on the runway while aircraft is approaching for landing in day, night,

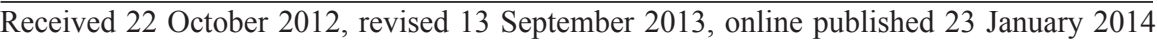


and foggy conditions. The airport assumed in present study is a regional airport where only one runway exists for operations.

\section{METHODOLOGY}

Figure 1 shows the flow chart of two methodologies for runway/obstacle detection proposed in this paper. First runway area is detected either by edge detection technique or by template matching technique. Once the runway area is detected subsequently for detecting obstacles on runway (runway incursions) edge detection technique is applied.

The first step in runway detection is to locate the runway area in an image taken from the sensors onboard the aircraft from approximately 3 nautical miles.

\subsection{Runway Search Area}

In case of straight and steady approach to landing scenario, pilot generally aligns the aircraft with the runway centre line and start descending. In such cases runway will always appear somewhere in the centre of the image and below the horizon. Therefore, for runway detection the search space in a given image is restricted to below the horizon.

\subsubsection{Horizon Detection}

In principle, horizon separates sky and earth surface through a straight horizontal line during level flight. In case of EO camera the colour image is first converted into gray level image. In the converted gray level image the sky and ground will have different gray levels. Using 'Gray Level Thresholding' horizon is detected from the converted gray level image as shown below.

Assuming size of the image I is M (rows) by N (columns), following equation is used to detect the horizon line

$$
I_{d}(i-1, j)=I(i, j)-I(i-1, j) \quad(2 \leq i \leq M \& 1 \leq j \leq N)
$$

where, size of $I_{d}$ matrix will be $M-1$ by $N$.

The row and column indices of horizon line are computed by comparing every element of $I_{d}$ for $j^{\text {th }}$ column against predetermined threshold which is obtained by trial and error method and retaining those indices at which element value exceeds the threshold. After getting row index for every column, the row coordinate of horizon line is computed by taking mean value of all row indices. Figure 2 shows the sequences of horizon detection process using simulated EO colour image in the flight simulator.

\subsubsection{Runway Search Area}

Once horizon is detected, runway search is restricted below horizon. As shown in Fig. 1, two methods are used to select runway search area below the horizon.

\subsubsection{Method 1}

\subsection{Initial Runway Search Area}

For the detection of runway it is assumed that aircraft while approach for landing is almost aligned to centre of runway. Once aircraft is aligned to the runway, then runway will be expected to be somewhere in the center of image and will start appearing below horizon. Hence runway search area is kept below horizon and extends from center of image to left and right corners as shown in Fig. 3(a). Once initial search area is selected then canny edge detection ${ }^{10}$ followed by Hough transform ${ }^{11-13}$ techniques are applied on the selected area to detect runway area (Fig. 3(b)).

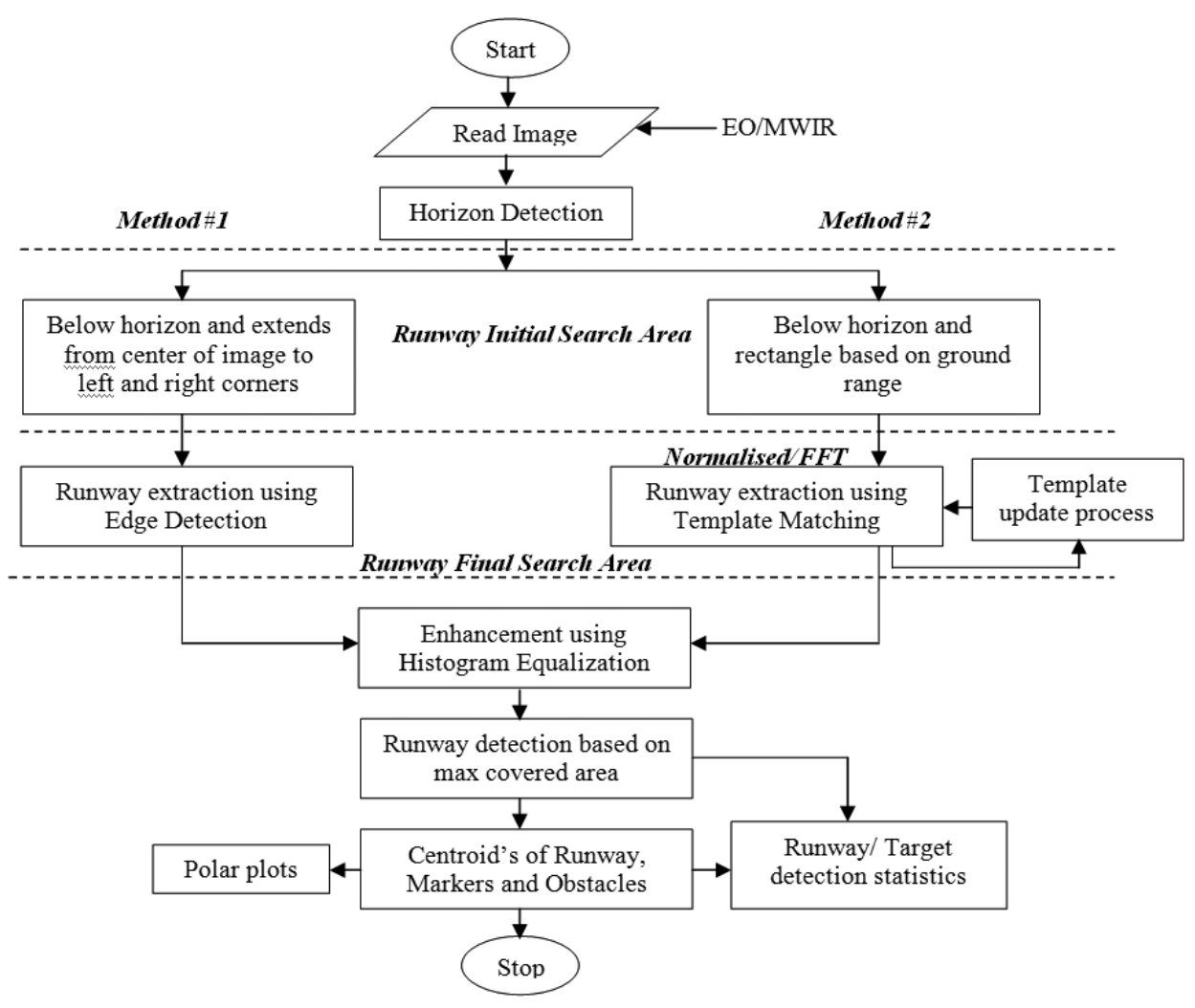

Figure 1: Flow chart of proposed methodologies. 


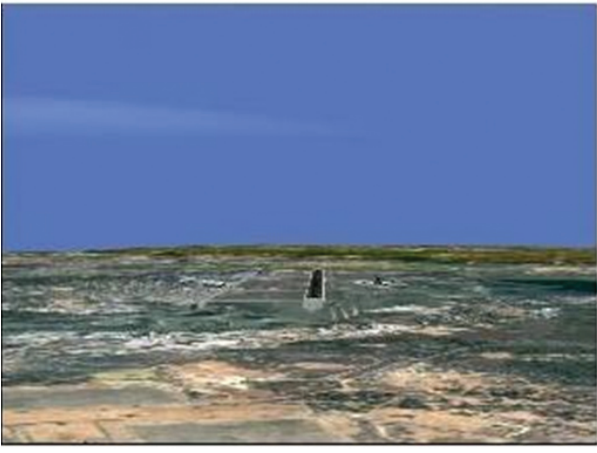

(a)

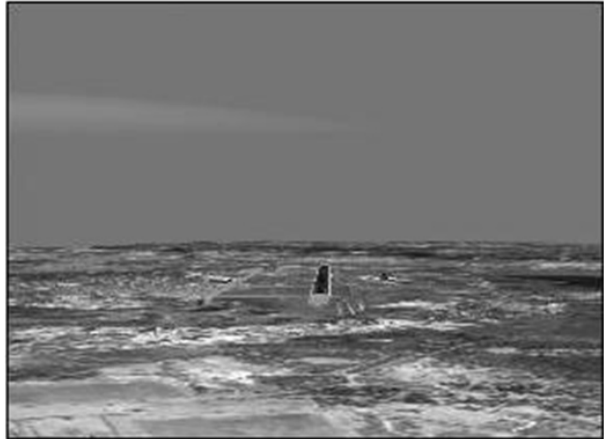

(b)

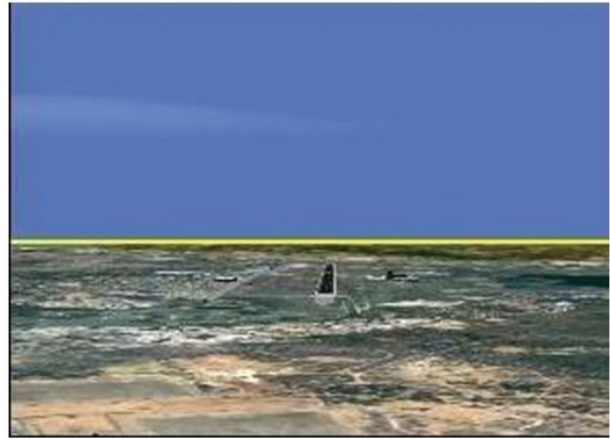

(c)

Figure 2. Horizon detection (a) Original image, (b) Gray level image and (c) Image with Horizon line.

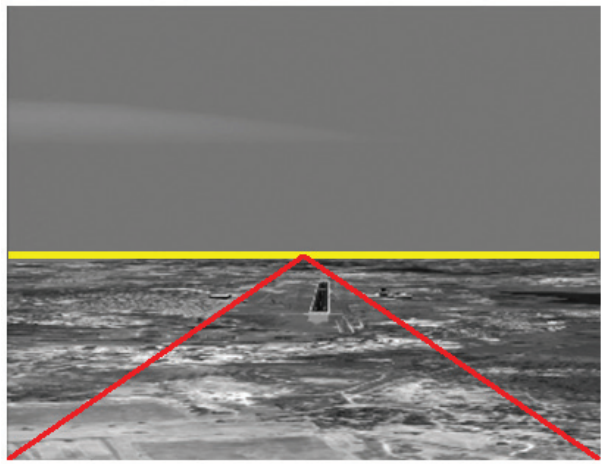

(a)

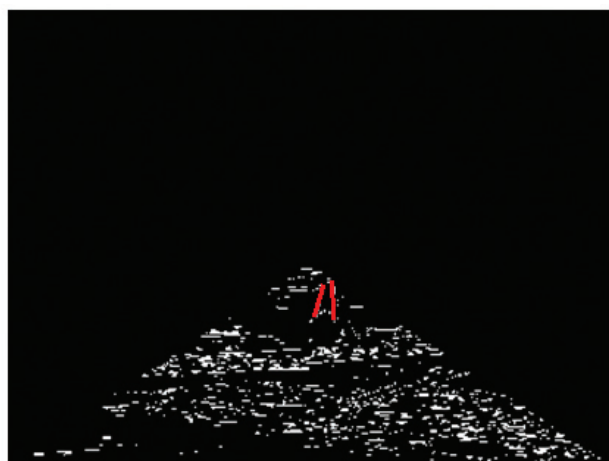

(b)

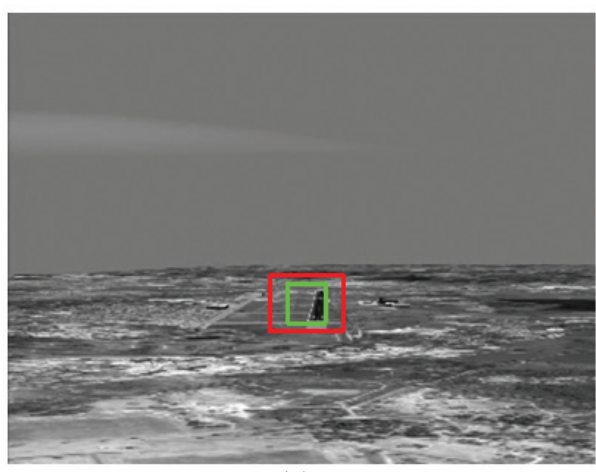

(c)

Figure 3. Selection of runway search area by method 1 (a) Initial search area, (b) Runway edge detection and (c) Final search area.

\subsection{Final Search Area}

Red markers shown in Fig. 3(b) indicate the coordinates of runway edges detected after applying Hough transformation. Once runway lines are detected, square is drawn around them based on their length and slope as shown in Fig. 3(c) (green). In order to consider adjacent taxi region the square area is extended to left and right sides by half of the magnitude of width of square. Similarly, square is extended up and down by one fourth of the magnitude of length of square (see red rectangle in Fig. 3(c)). Extension in horizontal is taken more than along vertical because taxiway in general will be located on the sides of runway.

\subsubsection{Method 2}

\subsection{Initial Runway Search Area}

In this method once horizon is detected, runway search is restricted below horizon within a rectangular search area extended by approximately half $\mathrm{km}$ to the left and half $\mathrm{km}$ to the right from center of the detected horizon, and extended approximately $3 \mathrm{~km}$ below the horizon (along runway) based on computed ground range of the aircraft from the runway threshold (approach end of the runway) and horizontal field of view (HFOV) of EO/MWIR camera. The ground range is computed using on-board global positioning system (GPS) data and runway threshold location available in WGS84 coordinates.

Let the image size generated by onboard EO/MWIR sensor be $\mathrm{M} \times \mathrm{N}$ (Fig. 4(a)) and $M_{1}$ is the row value of the image at which horizon line is detected, $\theta$ is the $\operatorname{HFOV~}\left(36^{\circ}\right)$ of EO/MWIR sensor, $\mathrm{R}$ is the ground range of aircraft from the runway threshold, $R_{L}(3 \mathrm{~km})$ and $R_{w}(1 \mathrm{~km})$ are the length and width of the runway search area.

The coordinates $\left(X_{1}, Y_{2}\right),\left(X_{2}, Y_{2}\right),\left(X_{3}, Y_{3}\right)$ and $\left(X_{4}, Y_{4}\right)$ of the initial search area (rectangle with solid line in Fig. 4(b)) are computed as follows:

$$
\begin{aligned}
& x_{2}=x_{1}=(N / 2)+\left(R_{w} /(2 * \Delta x)\right) \\
& y_{4}=y_{1}=M_{1} \\
& y_{3}=y_{2}=y_{1}+\left(R_{L} / \Delta y\right) \\
& x_{4}=x_{3}=(N / 2)-\left(R_{w} /(2 * \Delta x)\right)
\end{aligned}
$$

where, $\Delta \mathrm{x}$ and $\Delta \mathrm{y}$ are the distances per pixel in horizontal and vertical direction on the image respectively which are computed as follows:

$$
\begin{aligned}
& \Delta x=\frac{2 *\left(R+R_{L}\right) * \tan \left(\frac{\theta}{2}\right)}{N} \\
& \Delta y=\frac{\left(R+R_{L}\right)}{\left(M-M_{1}\right)}
\end{aligned}
$$

It can be observed from Eqn. (3) that $\Delta \mathrm{x}$ and $\Delta \mathrm{y}$ are proportional to range $R$ thereby enabling runway search area in pixel $^{2}$ to change dynamically (although its equivalent physical area in $\mathrm{km}^{2}$ will remain constant).

\subsection{Final Search Area}

If the aircraft approach path is aligned with the runway center line then the runway will be almost at the centre of the 

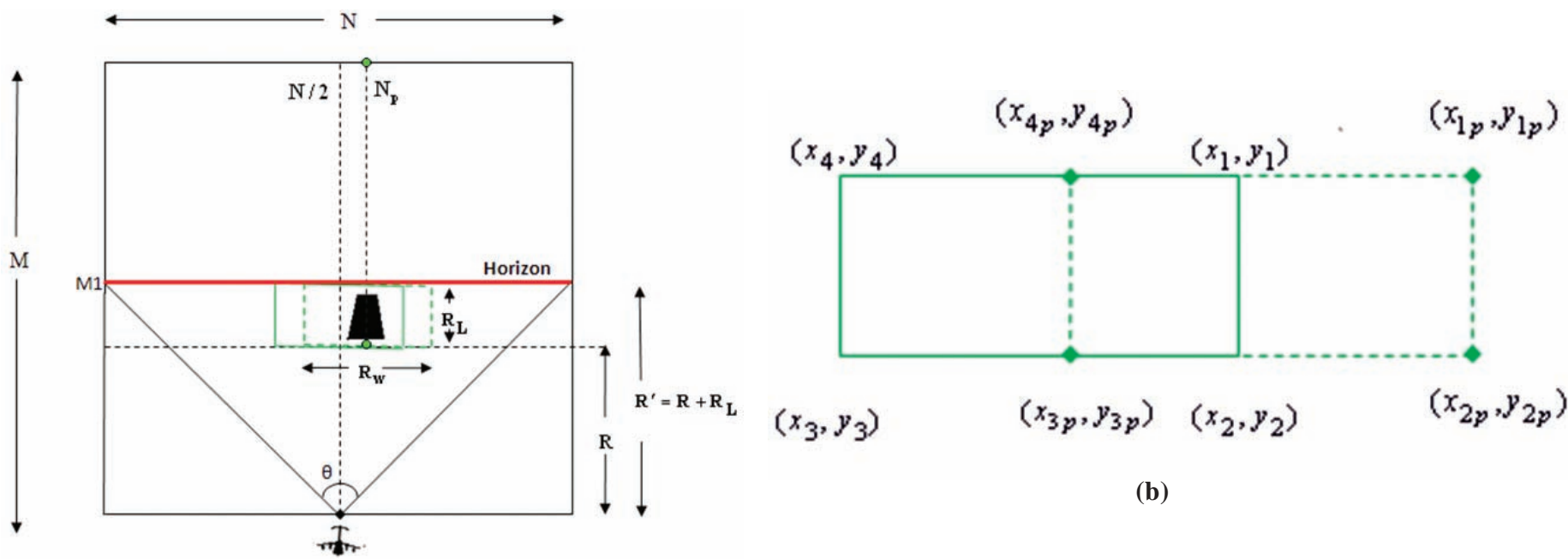

(a)

Figure 4. Geometry of runway search area.

rectangular search area as desired. But if aircraft approach path is not aligned with runway center line, the runway may not be at the centre of the rectangular search area. Then in such cases runway search area will be required to shift based on the computed offset between aircraft approach path and runway centerline as shown in Fig. 4(b). The rectangle with continuous solid line shown in Fig. 5 indicates the initial search area when aircraft approach path is not aligned with runway centerline and the rectangle with dotted line indicates the desired runway search area which is symmetrical about runway centerline.

Once the final runway search area on the image is selected, that portion of the image is cropped out for further processing to detect runway and obstacles on runway.

\subsection{Automatic Runway Detection}

Runway detection from the selected runway search area is carried out using two different methods ( Fig. 1) namely edge detection and template matching techniques. With EO images both techniques are applied and with MWIR images only edge detection technique is used. Following subsections details the two techniques for runway detection.

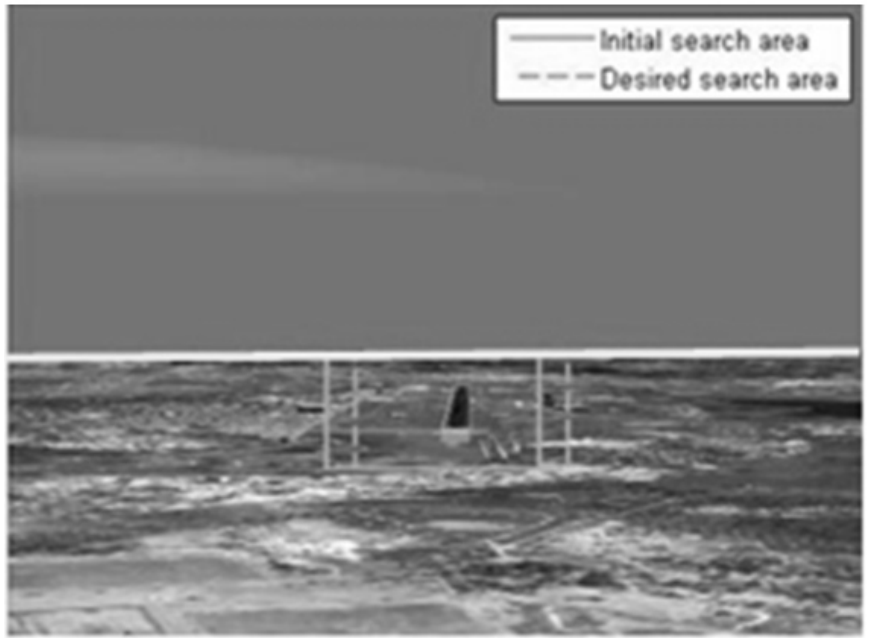

Figure 5. Detection of final runway search area.

\subsubsection{Runway Detection by Edge Detection Technique (Method 1)}

On the selected search area of the image (Fig. 6(a)), canny edge detection technique ${ }^{10}$ is again applied to get binary digital image with clear edges featuring runway and surrounding area. As seen on image captured by onboard camera of approaching aircraft, runway usually appears as trapezoidal with two horizontal parallel lines and other two lines of same length (runway edges) with symmetrical in nature. To detect the runway edges Hough transform ${ }^{11-13}$ is applied on binary image while searching maximum of two peaks with each peak corresponding to runway edges (Fig. 6(b)).

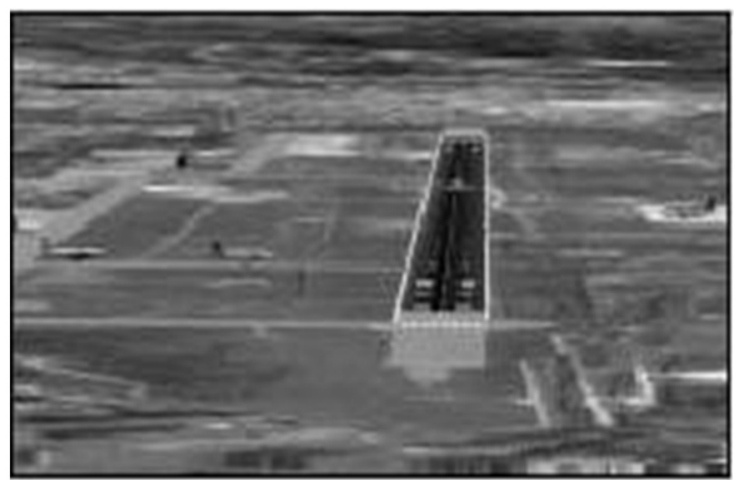

(a)

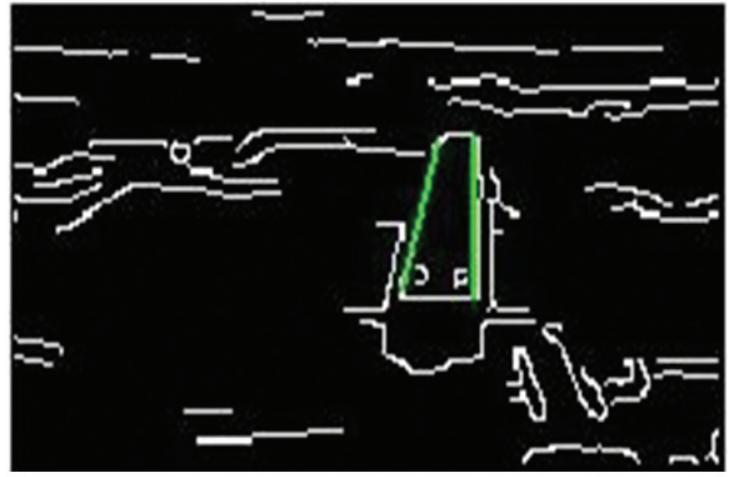

(b)

Figure 6. Hough transform on selected search area (a) Runway searched area, and (b) Detected runway edges. 


\subsubsection{Runway Detection by Template Matching (Method 2)}

In template matching technique cross correlation method ${ }^{14}$ is used for the detection of runway. In this technique the image template of the runway specific to particular airport is correlated with the cropped image and cross-correlation at each and every pixel is calculated. The pixel position at which maximum value of cross-correlation occurs is recognized as the Region of Interest (ROI) which is the likely position of the runway on the image around which runway is extracted with image size same as that of template.

\subsubsection{Template Matching using Normalized Cross- Correlation}

let $\mathrm{f}(\mathrm{x}, \mathrm{y})$ denotes the intensity value at the point $(x, y)$ in the cropped image of the size M x N (Fig. 7(a)). The runway pattern is represented by a runway template $t(x, y)$ of the size $\mathrm{m} \times \mathrm{n}$ (Fig. 7(b)). A common way to calculate the position of the runway pattern in the cropped image is by computing normalized cross-correlation value $\gamma$ at the point $(u, v)$ of cropped image and the runway template which has been shifted by $u$ steps in $\mathrm{x}$-direction and $v$ steps in y-direction.

The normalized cross correlation is computed using following equation:

$$
\gamma(u, v)=\frac{\sum_{x, y}\left(f(x, y)-\bar{f}_{u, v}\right)(t(x-u, y-v)-\bar{t})}{\sqrt{\sum_{x, y}\left(f(x, y)-\bar{f}_{u, v}\right)^{2} \sum_{x, y}(t(x-u, y-v)-\bar{t})^{2}}}
$$

where, $\bar{t}$ denotes the mean intensity value of template, similarly $\bar{f}_{u, v}$ denotes the mean intensity value of cropped image within the area of the template $t(x, y)$ shifted to $(u, v)$ which is calculated as

$$
\bar{f}_{u, v}=\frac{1}{m n} \sum_{x=u}^{u+m-1 v+n-1} f(x, y)
$$

The size of normalized cross-correlated image will be $\left(\mathrm{M}^{\prime}-\mathrm{m}+1\right) \times\left(\mathrm{N}^{\prime}-\mathrm{n}+1\right)$. The bright spot in Fig. 7(c) indicates the maximum correlation value. Region of interest (ROI) that is runway region is formed (as explained in section 2.2.2) using point $(u, v)$ at which cross-correlation is maximum (Fig. 7(d)).

\subsubsection{Template Update}

To align the aircraft approach path to the runway centerline, pilot may need to maneuver the aircraft and this may cause changes in runway perspective as seen through the onboard camera. Therefore, it is essential to update the runway template with previously matched runway in order to get consistent template matching with the next image frame during landing. In the present study the decision to update the template is based on comparison of image histogram of current runway template and the currently detected runway on the cropped image as explained in the following steps.

a) Compute the histograms of both the runway template and detected runway on the cropped image as $h_{t}$ and $h_{r}$ respectively.

b) Find the difference between the histograms $\left(H=h_{t}-h_{r}\right)$

c) Compute the normalized auto correlation sequence of the difference $\mathrm{H}$

d) Find the number of times the normalized autocorrelation $\begin{aligned} & \text { exceed the theoretical bounds } \\ & \text { (out of } 256 \text { gray level samples) }\end{aligned}$
given by $\mathrm{Th}= \pm \frac{1.96}{\sqrt{256}}$

If the percentage of normalized autocorrelation out of bounds is less than 6 per cent then the runway template is updated with the detected runway on the cropped image else current runway template is retained for next frame. In case of EO images taken at low light condition the template is updated only if the percentage of normalized autocorrelation out of bounds is less than 1 per cent. This is because the gray level variation (after converting to gray level image) of the EO image taken at low light condition is much less than the gray level variation in the EO image taken at normal light condition.

It is observed that in case of EO images both the edge detection technique as well as template matching technique is suitable for detecting runway in the search area. However, it was found that with template matching technique the uncertainty of detecting runway is less as compared to edge detection technique. In MWIR images, template matching is not working satisfactorily due to insufficient information on runway features. Hence it is decided to use edge detection technique for runway detection in MWIR images and template matching technique for runway detection in EO images.

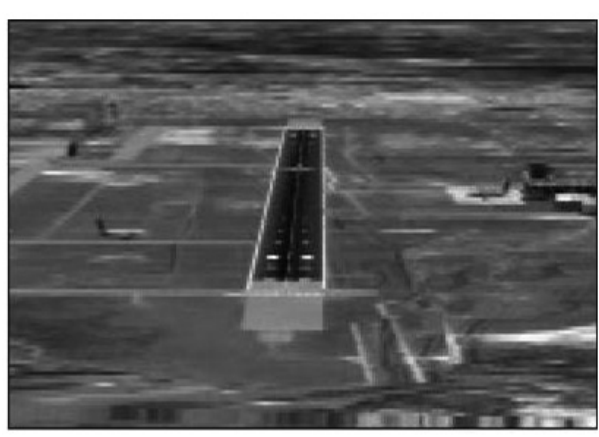

(a)

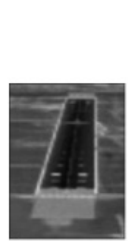

(b)

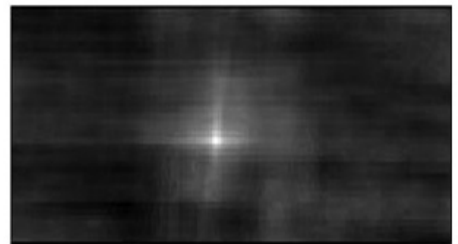

(c)

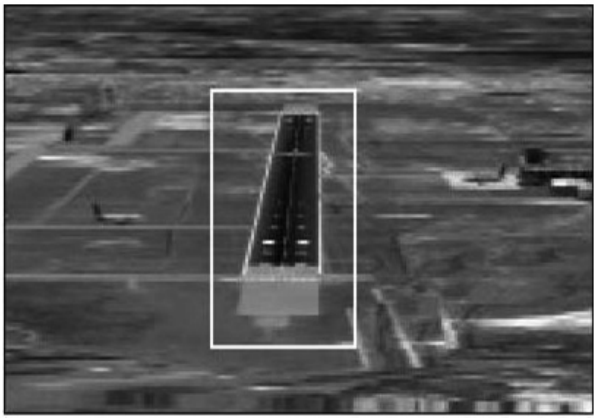

(d)

Figure 7. Runway detection using template matching (a) Cropped image, (b) Runway template, (c) Cross-correlation and (d) Detected runway. 


\subsection{Automatic Runway Obstacle Detection}

Target detection is the process of localizing those areas in the image where a potential target is likely to be present. Automatic target detection algorithm should be implicitly capable of rejecting the clutter from image background and then detecting targets by minimizing the probability of false alarm and maximizing the probability of target detection. In case of runway obstacle detection, the detection technique should be capable of detecting the distant objects (about 3 nautical miles) in order to give the pilot enough response time to take appropriate safety action.

Before runway obstacle detection, the cropped image with runway is subjected to image enhancement. The image enhancement technique adopted for normal light conditions (day time with clear visibility) and low light conditions (night time or low visibility conditions) are different, because in case of low light conditions runway lights generally will be 'ON' and they would help to find out runway edges but the edges does not appear as closed boundary. For example, in normal light conditions image enhancement is done by histogram equalization (HE) technique ${ }^{16}$ and in low light conditions image enhancement is done by de-blurring (2D filtering) ${ }^{17}$. It is observed from image histogram of cropped images that in case of low light conditions more number of pixels is having graylevel value less than 50 compared to normal light conditions. This fact is used to automatically select appropriate image enhancement technique of cropped image.

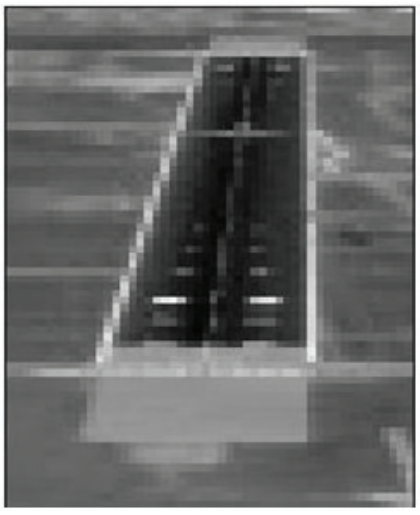

(a)

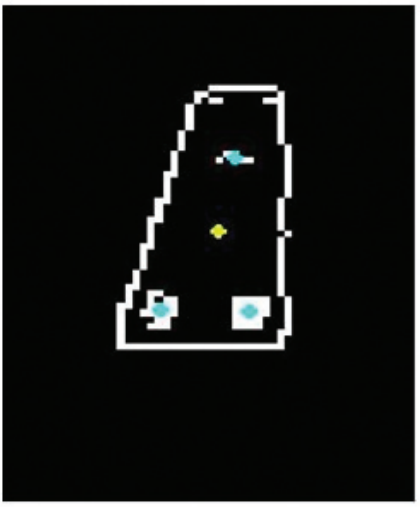

(e)

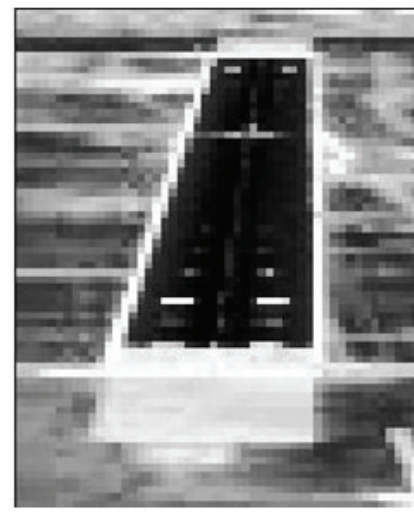

(b)

\subsubsection{Normal Light Condition}

Figure 8(a) shows the cropped image of EO sensor with detected runway during normal light condition. This cropped image is subjected to image enhancement using HE to bring the runway edges clearer as compared to surrounding regions (see Fig. 8(b)). To extract runway and obstacles on runway, canny edge detection technique is applied on enhanced image. Figure 8(c) shows the image with edges detected. Figure 8(d) shows the edges superimposed on cropped image. Dotted lines shows edges which are not closed. Along with runway edges there can be some edges which forms closed curves. Out of those closed curves only curve having maximum area is identified as runway as shown by solid line in Fig. 8(d).

Figure 8(e) shows the binary image of filtered runway. ' + ' symbol shows centroid of runway and ' $*$ ' symbol show centroid of detected targets on runway. The two detected targets near the approach end of the runway are identified as runway markers as they are static with respect to runway centroid and are symmetrical about runway centerline. The other target detected on the runway has been declared as obstacle due to its shape which cannot possibly be a runway marker.

Following equations are used to compute range (in pixels) and bearing (in deg) of detected targets on runway with respect to runway centroid and plotted in polar form (Fig. 8(f)) to indicate whether obstacle is moving or static, approaching or moving away from landing aircraft:

$$
\text { range }=\sqrt{\left(C_{x}^{O}-C_{x}^{i}\right)^{2}+\left(C_{y}^{o}-C_{y}^{i}\right)^{2}} \quad \text { in pixels }
$$

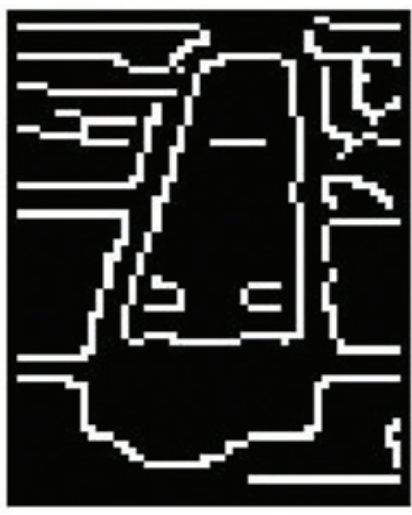

(c)

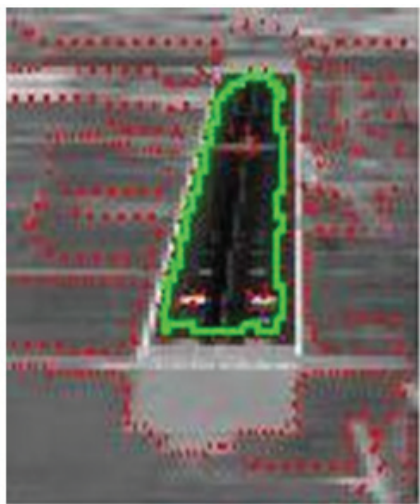

(d)

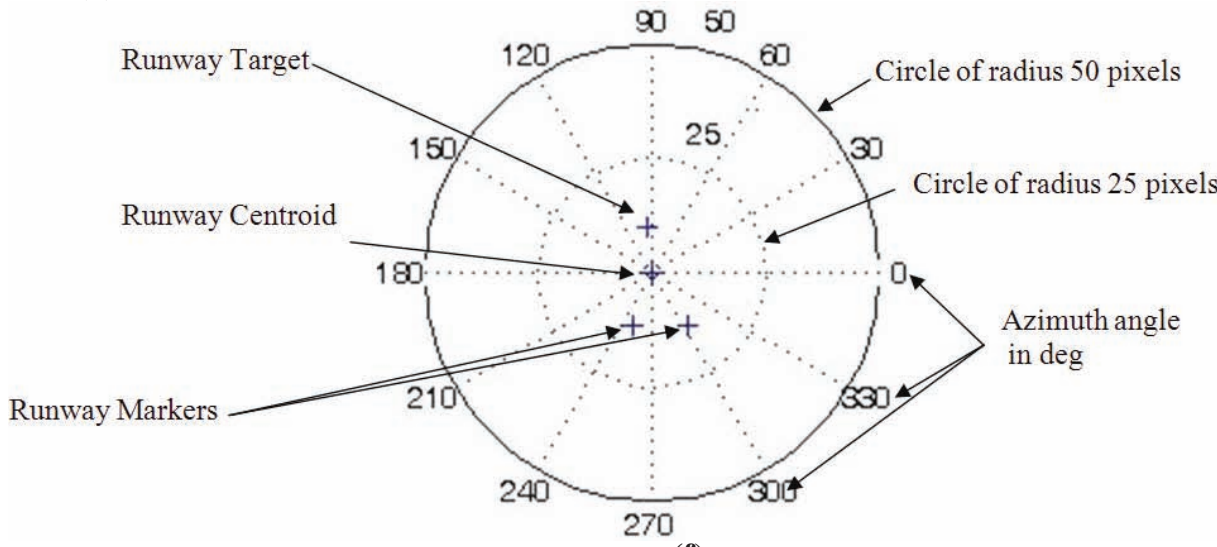

(f)

Figure 8. Runway and obstacle detection in normal light condition (a) Cropped image with runway, (b) Enhanced image, (c) Edge detected (binary), (d) Detected edges (labeled), (e) Binary image of runway with markers and target and (f) Location of detected targets and markers w.r.t runway centroid. 


$$
\text { bearing }=\tan ^{-1}\left[\frac{\left(C_{y}^{O}-C_{y}^{i}\right)}{\left(C_{x}^{O}-C_{x}^{i}\right)}\right] \text { in degrees }
$$

where, $\left(C_{x}^{0}, C_{y}^{0}\right)$ is centroid of runway and $\left(C_{x}^{i}, C_{y}^{i}\right)$ is the centroid of $\mathrm{i}^{\text {th }}$ object (includes runway, obstacles on runway and runway markers).

\subsubsection{Low Light Condition}

As mentioned before, in case of low light conditions runway lights help to find runway edges. Figure 9(a) shows the cropped image from EO camera in low light condition with detected runway where it can be seen that runway edges appears to be blurred. It is observed that by applying edge detection directly on cropped image with blurred edges results in undesirable dual runway edges on both sides. Therefore, cropped image is first de-blurred as shown in Fig. 9(b). The other problem encountered in this case is runway does not appear as closed boundary to declare it as runway. Therefore, Hough transform is applied on the de-blurred image to detect the four coordinates of runway by detecting the lines representing the runway, and then simply join all four coordinates to close the boundary as shown in Fig. 9(c). Figure 9(d) shows edges in solid and dotted lines. Figure 9(e) shows the detected runway. Figure 9(f) shows the polar plot with centroid of runway as origin. In this case, as there are no targets detected on the runway, no targets are appearing in polar plot.

\section{DATA SIMULATION AND VALIDATION}

To evaluate proposed technique for detection of runway and runway incursions, simulated image data of EO colour camera on board a fighter aircraft approaching/landing at Bangalore HAL airport during day with clear visibility condition is generated using the engineer-in-loop simulator $(\text { ELS })^{18}$. Similarly simulated data of MWIR sensor on board a regional transport aircraft approaching or landing at HAL airport during day and night with fog CAT II visibility condition is generated using augmented engineering environment (AEE) research flight simulator (CAE Simfinity integrated procedures trainer $\left.{ }^{19}\right)$. Approach for landing is simulated with static as well as moving obstacle(s) placed on the runway. In every case, the detection is carried out starting at a distance of 3 nautical miles during approach for landing. To restrict the paper length only following two case studies have been presented.

\subsection{Case 1: Single Moving Obstacle in Normal Light Condition}

Figure 10(a) shows binary image of detected runway, the runway markers, and the obstacle on the runway in different video frames of on-board EO camera. It can be observed from this sequence of video frames that obstacle is moving towards landing aircraft. Figure 10(b) shows the location of obstacle and runway markers in the form of polar plot for the entire video. It can be observed from the polar plot that the obstacle

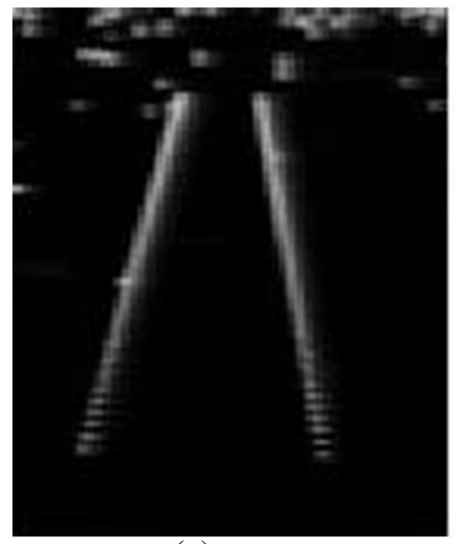

(a)

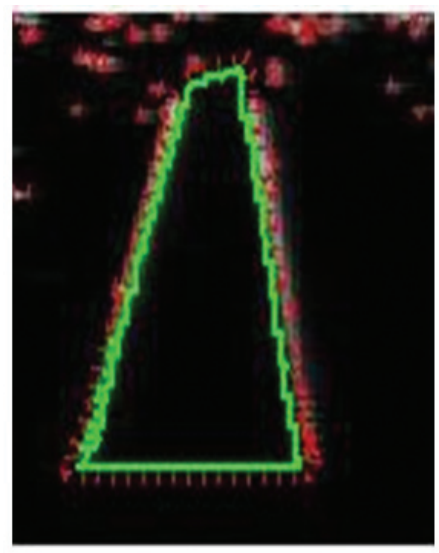

(d)

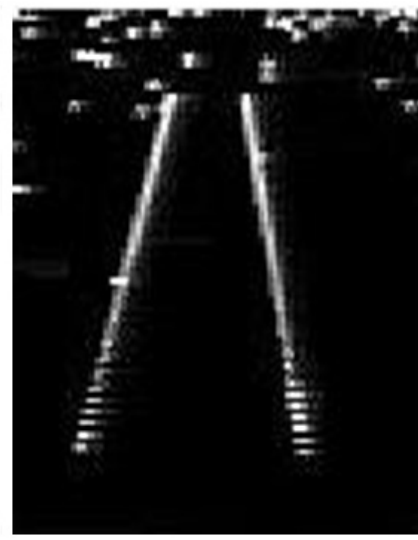

(b)

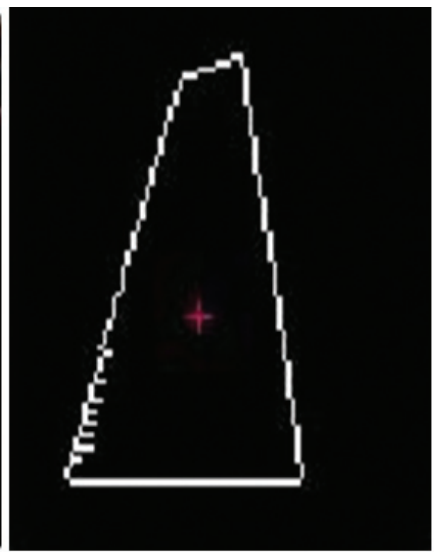

(e)

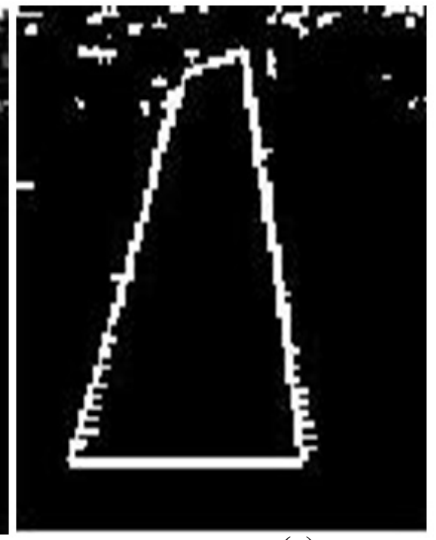

(c)

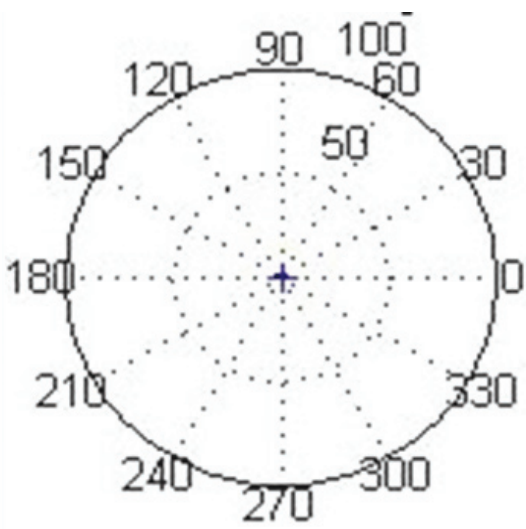

(f)

Figure 9. Runway and obstacle detection in low light condition (a) Cropped image with runway (b) De-blurred image, (c) Edge detected (binary), (d) Detected edges (labeled), (e) Binary image of runway with markers and target and (f) Location of detected markers and targets on runway. 
is moving on the left side of runway and towards the centroid of runway. It can also be observed from Fig. 10(b) that runway markers appears to be moving on polar plot though they are static because as aircraft approach towards runway the camera view angle changes and these markers start appearing larger and their centroid changes (see Fig. 10(a)).

\subsection{Case 2: Multiple Static Obstacles in Night Foggy Condition with MWIR Camera}

Figures 11(a) and 11(b) show the simulated images from on-board EO and MWIR cameras during approach for landing initiated from 3 nautical miles from runway threshold in night time under fog CAT II visibility condition. Three different aircrafts are placed at different locations along the runway as obstacles. Figure 11(c) show the binary images of detected runway and runway obstacles at different video frames from the MWIR camera. Figure 11(d) shows the location of obstacle with respect to runway centroid in the form of polar plot for entire video.

\section{ANALYSIS OF DETECTION TECHNIQUES \\ 4.1 Statistics of Runway and Runway Incursion \\ Detection}

This paper presents a novel method for detection of runway and runway incursions using onboard EO camera and MWIR sensors starting from a distance of approximately 3 nautical miles while the aircraft is approaching for landing. Therefore, effectiveness of the technique depends on the success rate of runway detection from 3 nautical mile distance. Table 1 gives the statistics of runway detection, miss detection and false alarm
FRAME : 101
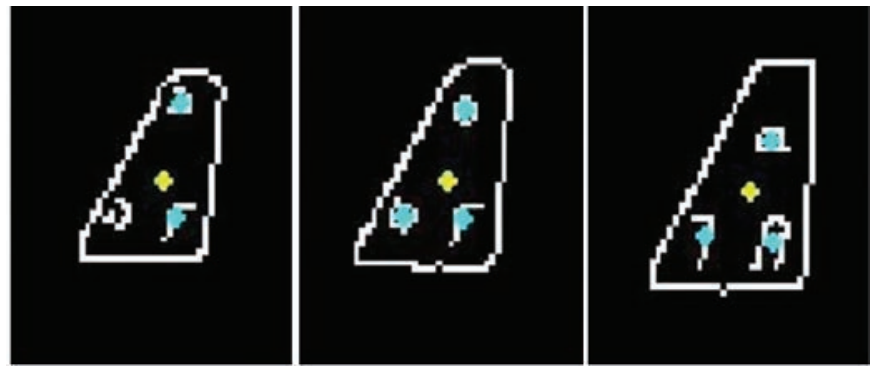

(a)

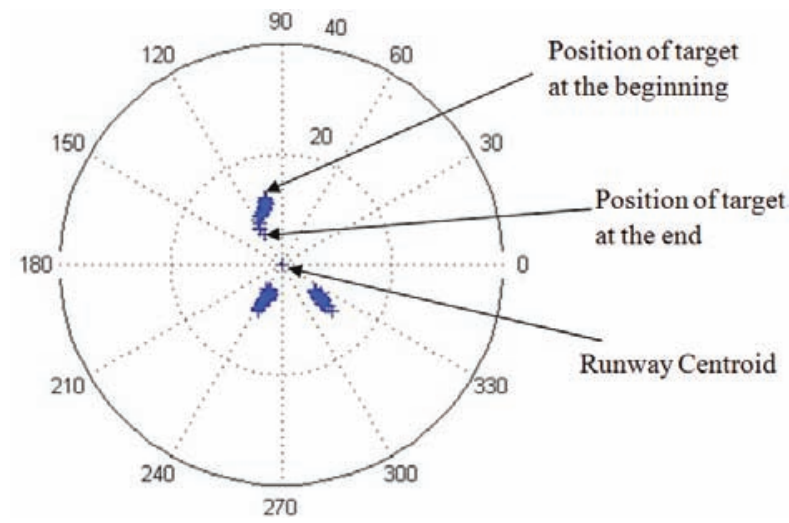

(b)

Figure 10. (a) Binary image of runway with runway markers and moving obstacle and (b) Location of runway markers and obstacle with respect to runway centroid.

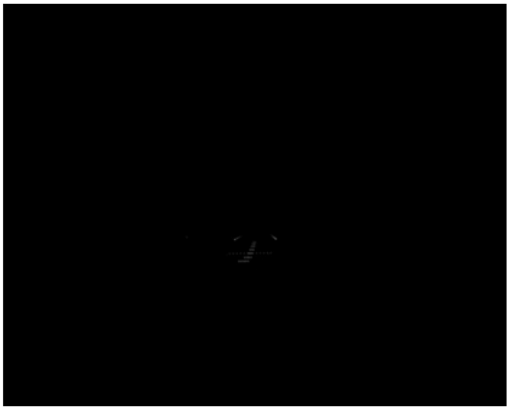

(a)

FRAME : 15

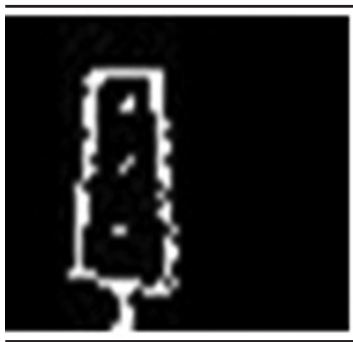

FRAME : 230

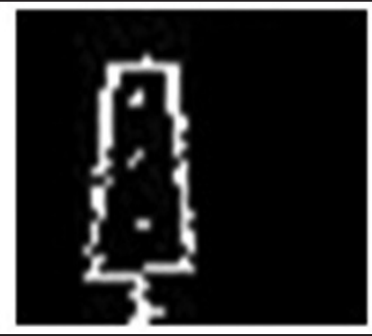

(c)

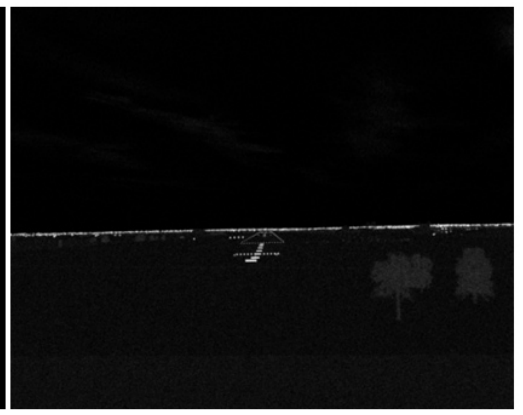

(b)

FRAME : 800

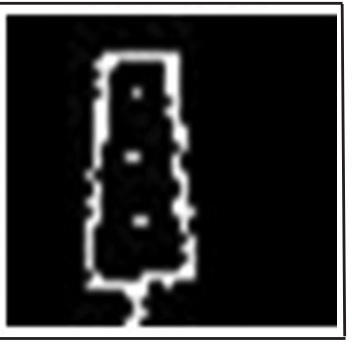

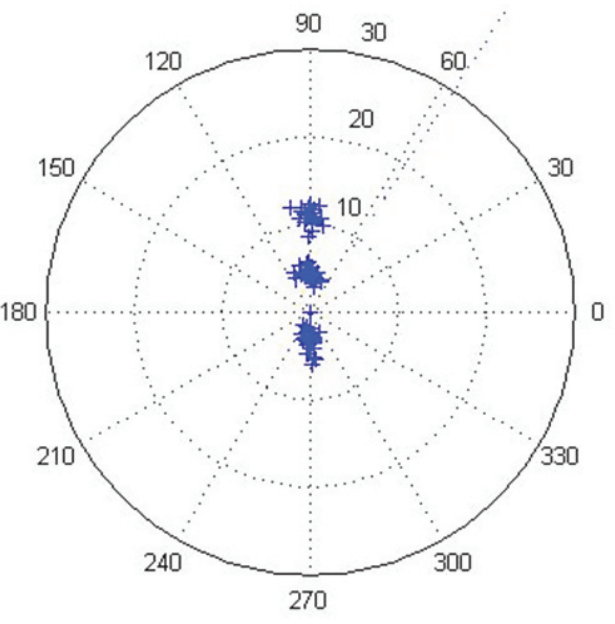

(d)

Figure 11. (a) Image from on-board EO camera in fog CAT II visibility in night, (b) Image from on-board MWIR camera in fog CAT II visibility in night, (c) Binary Images of runway with obstacles from MWIR sensor and (d) Location of obstacle with respect to runway centroid. 
for three different case studies. As mentioned in section 3, test data is simulated with EO camera onboard a fighter aircraft and MWIR sensor onboard a regional transport aircraft and hence the number of video frames analyzed for runway detection in each case is different. Further, in AEE flight simulator ground range measurement was not available and hence with MWIR sensor template matching technique (Method 2 shown in Fig. 1) could not be used for runway detection.

From the statistics of the Table 1, it can be observed that the probability of runway and runway incursion detection is relatively more in case of EO camera under normal light condition compared to MWIR sensor under low light condition. Results also show that the template matching technique is relatively more efficient than edge detection technique in detecting the runway and runway incursions. In case of MWIR sensor, since only edge detection technique was possible to use, the probability of false alarm is more than probability of runway detection. With template matching technique probability of runway detection can be expected to be improved.

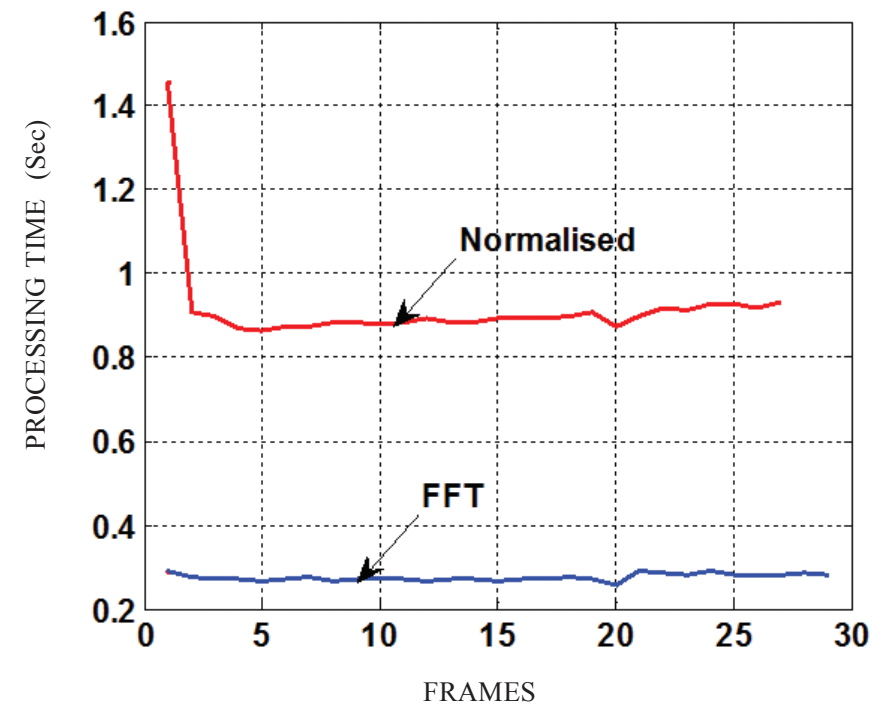

Figure 12. Processing time per frame.

Table 1. Statistics of runway and runway incursion detection

\begin{tabular}{rlclccccc}
\hline $\begin{array}{l}\text { Case } \\
\text { study }\end{array}$ & $\begin{array}{l}\text { Visibility } \\
\text { condition }\end{array}$ & $\begin{array}{l}\text { Sensor/ } \\
\text { Camera } \\
\text { used }\end{array}$ & $\begin{array}{l}\text { Detection } \\
\text { method }\end{array}$ & $\begin{array}{l}\text { No. of } \\
\text { frames } \\
\text { analyzed }\end{array}$ & $\begin{array}{l}\text { No. of frames } \\
\text { with runway } \\
\text { detected }\end{array}$ & $\begin{array}{l}\text { No. of frames } \\
\text { with runway } \\
\text { not detected }\end{array}$ & $\begin{array}{l}\text { No. of frames } \\
\text { with false } \\
\text { alarm }\end{array}$ & $\begin{array}{l}\text { No. of frames with } \\
\text { target detected on } \\
\text { runway* }\end{array}$ \\
\hline 1 & Normal light & EO & Method1 & 320 & $117(37 \%)$ & $145(45 \%)$ & $58(18 \%)$ \\
2 & Normal light & EO & Method2 & 320 & $183(57 \%)$ & $89(28 \%)$ & $48(15 \%)$ \\
3 & Low light & MWIR & Method1 & 805 & $101(13 \%)$ & $490(60 \%)$ & $214(27 \%)$ \\
\hline
\end{tabular}

${ }^{* *}$ This is a subset of 'No. of frames with runway detected' (column 6)

\subsection{Template Matching using Fast Fourier Transform}

It is often observed that template matching in spatial domain (normalized cross correlation technique mentioned in section 2.2.2.1) is computationally slow which affects the output frame rate significantly. To overcome this problem template matching is tried out in frequency domain using Fast Fourier Transform $(\mathrm{FFT})^{20}$. In frequency domain the cross correlation is computed using following steps:

- The cross correlation of cropped image $f(x, y)$ and runway template $t(x, y)$ in frequency domain is computed as:

$$
C C(p, q)=F(p, q) \cdot T^{*}(p, q)
$$

where, $F(p, q)$ is the FFT of $f(x, y)$ and $T^{*}(p, q)$ is the complex conjugate of FFT of template $t(x, y)$.

- The inverse FFT of $C C(p, q)$ gives cross correlation in time domain which is used for template matching

- The position at which maximum value of cross-correlation occurs is recognized as the position of the runway around which ROI is formed in the cropped image.

Figure 12 shows the comparison of processing time by FFT (frequency domain) and normalized cross correlation (spatial domain) techniques.

\section{CONCLUSIONS}

Techniques to detect runway and runway incursions (obstacles on runway) using Electro-Optical (EO) colour camera and Medium Wave Infrared (MWIR) sensor on-board the aircraft during approach for landing have been proposed, implemented and demonstrated. For runway detection, canny edge detection technique with Hough transform and template matching technique are proposed. Once runway is detected, obstacles on the runway are detected using edge detection technique. The detected runway obstacles are presented pictorially in polar plot to indicate their location on runway (in pixel coordinates w.r.t. centroid of runway) and whether they are static or moving. The performance of the proposed techniques are evaluated in flight simulators with simulated images of EO and MWIR cameras on-board the aircraft during approach for landing at a distance of 3 nautical miles from runway threshold during day/night and in low visibility CAT II foggy conditions. Effectiveness of the techniques with statistics of runway detection, miss detection and false alarm for different case studies have been provided and discussed. Results show that the probability of runway and runway incursion detection is relatively more in case of EO camera under normal light condition compare to MWIR sensor under low light condition and the template matching technique is relatively more efficient than edge detection technique in detecting the runway and runway incursions. As part of future work the detection of runway incursions from real images of EO and MWIR is being planned. Also further research is planned to improve the probability of runway detection with MWIR sensor under low light condition. 


\section{ACKNOWLEDGEMENT}

Authors acknowledge the support extended by Flight Simulation group of NAL for carrying out this research with data generated on ELS and AEE flight simulators.

\section{REFERENCES}

1. Prinzel L.; Hughes, M., Kramer L. and Arthur J. Aviation safety benefits of NASA synthetic vision: Low visibility loss-of-control, runway incursion detection, and CFIT experiments. In the Proceedings of the human performance, situation awareness, and automation technology conference. Daytona Beach, FL, 2004.

2. Federal Aviation Administration. Runway Incursion severity trends at towered airports in the United States. FAA Runway Safety Report. June 2001.

3. Cheng-Hua Pai; Yu-Ping Lin; Gerard G. Medioni and Ray Rida Hamza. Moving object detection on a runway prior to landing using onboard infrared camera. In the IEEE conference on computer vision and pattern recognition: E-ISBN: 1-4244-1180-7/07, 2007.

4. Cynthia Archer; Joseph White and Robert Neece. Airborne FLIR sensors for runway incursion detection. In the Proceeding of SPIE: Enhanced and Synthetic Vision, 7328, 73280E, 2009.

5. Joseph, H. White; James, G. Haidt; Charles, L. Britt; Cynthia, Archer \& Robert, T. Neece. Fusion of airborne radar and FLIR sensors for runway incursion detection. In Proceeding of SPIE, 7458, 74580A-1, 2009.

6. Abdul, Rahiman M., Mahalekshmi, Monisha C.; Remya S, Manoj Kumar G and Rajasree M S. Automated object recognition and pattern matching analysis of runways using surface track data. In the International Conference on Electronics Computer Technology (ICECT), Kanyakumari, April 2011, pp. 46-51.

7. Yinwen Dong, Bingcheng Yuan, Hangyu Wang, and Zhaoming Shi. A runway recognition algorithm based on heuristic line extraction. In the International Conference on Image Analysis and Signal Processing (IASP), Hubei, October 2011, pp. 1-5.

8. Xiaobing Wang, Baokui Li and Qingbo Geng. Runway detection and tracking for unmanned aerial vehicle based on an improved canny edge detection algorithm. In the $4^{\text {th }}$ International Conference on Intelligent Human-Machine Systems and Cybernetics, Nanchang, Jiangxi (Chaina), August 2012, pp. 149-152.

9. Aytekin, Ö.; Zöngür, U \& Halici, U. Texture-based airport runway detection. IEEE Geosc. Remote Sensing Letters, 2012, 10(3), 471-475.

10. Canny, J. A computational approach to edge detection. IEEE Trans. Pattern Analysis Machine Intell. 1986, 8(6), 679-698.

11. Ballard, D. H. Generalizing the Hough transform to detect arbitrary shapes. Pattern Recognition, 1981, 13(2), 111122.

12. Richard, O. Duda \& Peter, E. Hart. Uses of the Hough transform to detect lines and curves in picture.
Communications ACM, 1972, 15(1), 11-15.

13. Jiann-Yeou, Rau \& Liang-Chien, Chen. Fast straight lines detection using hough transform with principal axis analysis. J. Photogrammetry Remote Sensing, 2003, 8(1), 15-34.

14. Kai, Briechle \& Uwe, D. Hanebeck. template matching using fast normalized cross correlation. In the Proceedings of SPIE: Optical pattern recognition. 2001, 4387, pp. 1-8.

15. James, V. Candy. Signal processing: The model-based approach, McGraw-Hill, 1987.

16. Zuiderveld, Karel. Contrast Limited Adaptive Histogram Equalization. Graphic Gems IV. San Diego: Academic Press Professional, 1994. pp. 474-485.

17. Rafel, C. Gonzalez \& Richard, E. Woods. Digital Image Processing, Addison Wesley, 1993.

18. http://www.nal.res.in/pages/theengineerintheloopsimulator.htm [Accessed on 12/09/2013]

19. http://www.cae.com/uploadedFiles/Content/ BusinessUnit/Civil_Aviation/Media_Centre/Document/ datasheet.cae.simfinity.pdf [Accessed on 12/09/2013]

20. Rafael, C. Gonzalez; Richard, E. Woods \& Steven, L. Eddins. Digital image processing using Matlab: Object recognition. 2008, pp.504-506.

\section{CONTRIBUTORS}

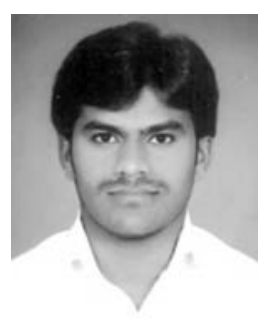

Mr Satish Kumar V received his BTech (Electronic and Communication Engineering) in 2008 and MTech (Signals and System Processing) in 2011 from Jawaharlal Nehru Technological University. He is currently working as a software engineer with QuestGlobal Technologies in Bangalore. His current research interest includes target detection, classification and image processing.

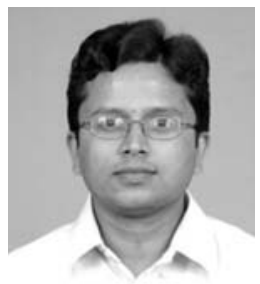

Dr Sudesh K. Kashyap obtained his ME (Electrical Engineering) from M.S. University of Baroda, Gujarat, in 1999 and $\mathrm{PhD}$ (Electrical and Electronics Engineering) from University of Mysore, Karnataka, in 2008. Presently, he is working at CSIRNational Aerospace Laboratories, Bangalore as Principal Scientist. His areas of interest include Kalman filtering, multi sensor data fusion, fuzzy logic, Bayesian theory, neural network and image processing.

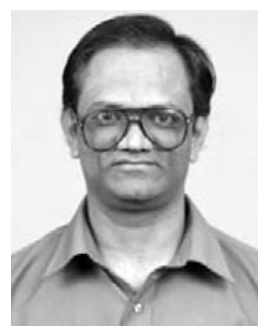

Mr N. Shantha Kumar obtained his MTech (Aerospace Engineering) from IIT Bombay in 1987. Presently he is working in Flight Mechanics and Control Division of CSIRNational Aerospace laboratories (NAL). His areas of interest include Multi sensor data fusion, Kalman filtering, target tracking and integrated enhanced and synthetic vision system. He is a life member of Aeronautical Society of India. 